\title{
ESTUDO DO CENÁRIO ENERGÉTICO PARA DESENVOLVIMENTO DE PROTÓTIPO DETECTOR DE FURTOS DE ENERGIA ELÉTRICA EMBUTIDOS EM PAREDES
}

Ane Caroline Teixeira da Paixão ${ }^{\text {; }}$ Juliana Maria Souza de Santana 2; Felipe Curcino Nascimento ${ }^{2}$; Ricardo Cerqueira Medrado ${ }^{2}$; Valmiro José Rangel Galvis ${ }^{2}$; Rosana Vieira Albuquerque ${ }^{3}$.

1 SENAI CIMATEC - Área de Eletrônica Embarcada, Instituto de Inovação em Automação; Salvador/BA; anecaroline teixeira@hotmail.com;

2 SENAI CIMATEC - Área de Eletrônica Embarcada, Instituto de Inovação em Automação; Salvador/BA;

${ }^{3}$ SENAI CIMATEC - Área de Gestão, Instituto SENAI de Inovação em Logística; Salvador/BA.

Resumo: O furto de energia se constitui como um dos maiores problemas para as empresas que detém a concessão da distribuição de energia elétrica no Brasil. Esta conduta delituosa traz como consequência o aumento da tarifa de energia, pois, segundo as normas da ANEEL, parte do prejuízo decorrente das perdas pode ser distribuído entre os consumidores regulares. Apesar do aumento da tarifa, as empresas de distribuição também amargam prejuízos decorrentes do ilícito, sendo preciso investir em novos métodos e equipamentos visando diminuir o furto. $O$ presente artigo discorre acerca de tecnologias para o desenvolvimento de um protótipo detector de desvios embutidos para identificar e comprovar os delitos sem a necessidade de realizar uma intervenção civil na propriedade do suspeito.

Palavras-Chave: Perdas; Energia; Furto; Detecção; Condutores.

\section{STUDY OF THE ENERGY SCENARIO FOR THE DEVELOPMENT OF A PROTOTYPE THAT DETECTS ELECTRICITY THEFT BUILT IN WALLS}

\begin{abstract}
Energy theft is one of the biggest problems for companies that hold concessions of electricity distribution in Brazil. This criminal conduct results in an increase in the energy tariff, because, according to ANEEL rules, part of the damage, resulting from the losses, can be distributed among regular consumers. In addition to the increase in the tariff, distribution companies also suffer losses due to the illicit, and it is necessary to invest in new methods and equipment to reduce theft. This paper discusses technologies for the development of a built-in deviation detector prototype to identify and prove offenses without the need for civil intervention on the suspect's property.
\end{abstract}

Keywords: Loss; Energy; Theft; Detection; Conductors. 


\section{INTRODUÇÃO}

A segmentação da cadeia produtiva dos sistemas elétricos de potência brasileiros se dá na geração, transmissão e distribuição de energia elétrica, correspondentes à produção, transporte e consumo da mesma. Dentro desses processos, há montantes de energia não contabilizados pelos sistemas de medição devido a existência de perdas, fracionadas em técnicas e não técnicas, caracterizadas pela diferença entre a energia que é produzida e a que de fato é comercializada.

As perdas técnicas, inerentes aos sistemas elétricos, correspondem à transformação de energia elétrica em térmica (Efeito Joule), perdas nos núcleos dos transformadores, entre outros. [1] Por sua vez, as Perdas Não Técnicas - PNT são consequência dos furtos feitos diretamente da rede por consumidores que não possuem vínculos contratuais com a concessionária (crime de furto segundo Art. 155 do Código Penal) [2] e de fraudes comumente feitas por meio de adulterações nos medidores por consumidores que são cadastrados no banco de dados da concessionária de energia correspondente (estelionato conforme Art. 171 do CP). [3]

Por consequência das perdas não técnicas, segundo dados da ANEEL, os prejuízos causados às concessionárias de energia chegaram, em 2017, à um montante de $R \$ 8,1$ bilhões, correspondendo a mais de 27 mil Gigawatts-hora (GWh), o suficiente para atender todas as 217 cidades maranhenses. Tais prejuízos são repassados aos consumidores, onerando as tarifas de energia elétrica. No caso da Light Serviços de Eletricidade S/A, concessionária de energia no Rio de Janeiro, os desvios na sua área de concessão impactam em uma elevação tarifária de cerca de $18 \%$. [4]

O furto de energia representa mais uma faceta do problema ético-social vivenciado cotidianamente no Brasil: a conivência com pequenos ilícitos e a certeza da impunidade. Embora os indicadores ilustram um cenário de maior ocorrência dos furtos em regiões economicamente menos favorecidas, o fenômeno não é exclusivo das comunidades de baixa renda. [5]

Os limites regulatórios de PNT são calculados sazonalmente nas revisões tarifárias. Dentro deste limite, todo o prejuízo decorrente das perdas pode ser repassado aos consumidores regulares, através da fatura de energia. [6] Entretanto, na maioria dos casos, o índice de PNT apurado é superior ao limite regulatório, e a concessionária de energia precisa absorver a parcela de prejuízo derivado do ilícito. [7]

Considerando esses fatos, as concessionárias buscam formas alternativas de detecção dos desvios, sendo as mais usuais: Análise de histórico de consumo de unidades consumidoras - Degraus de consumo, Localização e Data Mining. [8] Baseadas nessas análises, são direcionadas equipes para inspeções no imóvel dos suspeitos. Tais inspeções podem causar constrangimento ao consumidor, especialmente nos casos em que não é constatada nenhuma irregularidade.

O problema de pesquisa é caracterizado pela baixa assertividade nos processos de inspeção e a falta de provas do ilícito, acarretando em recorrência nos atos, e, por conseguinte, nos prejuízos às concessionárias. Diante dos fatos expostos, 
como poderia ser melhorada a baixa assertividade do atual processo de inspeção e detecção de fraudes?

Visando solucionar o problema descrito, foi proposto pelo projeto de pesquisa (PD-0382-0121/2018):

- O desenvolvimento de um sistema para detecção de desvios embutidos;

- A simplificação dos métodos de detecção;

- A geração de provas do ilícito.

Este artigo tem como objetivo geral apresentar os estudos das etapas preliminares de desenvolvimento de um novo equipamento voltado para identificação de fraudes embutidas. Tais estudos se basearam, principalmente, na análise dos métodos mais usuais de furtos, nos problemas encontrados nos métodos de detecção atuais quanto à comprovação do desvio, geração das provas do delito e testes dos dispositivos mais utilizados na identificação das fraudes.

O benefício esperado é a redução da impunidade dos fraudadores através de um novo sistema, mais simplificado, de identificação e comprovação de detecção. Com isso, o projeto visa reduzir o número de fraudes, resultando em benefícios tanto para as concessionárias quanto para os consumidores.

Este trabalho foi desenvolvido no âmbito do Programa de Pesquisa e Desenvolvimento Tecnológico do Setor de Energia Elétrica regulado pela ANEEL e as reflexões e resultados apresentados são fruto do projeto de Pesquisa e Desenvolvimento "Identificação de fraudes embutidas por sobreposição de camadas de imagens". Código ANEEL PD-0382-0121/2018, em execução pelo Senai CIMATEC e com conclusão prevista para agosto/2020, realizado com suporte financeiro da Light Serviços de Eletricidade.

\section{METODOLOGIA}

Através de pesquisas bibliográficas foram identificados os requisitos do protótipo a ser desenvolvido a partir da análise dos métodos mais usuais de desvios embutidos, além de estudos sobre as ações das concessionárias no processo que abrange da análise à detecção do possível furto de energia.

Posteriormente, para coleta dos dados referentes aos dispositivos, foram feitas pesquisas exploratórias, com finalidade de obter informações dos equipamentos já existentes no mercado e quais são os princípios de funcionamento destes. Com essas informações, torna-se possível saber quais funcionalidades e tecnologias serão úteis para desenvolvimento do protótipo.

Figura 1. Etapas Metodológicas das fases iniciais do projeto.

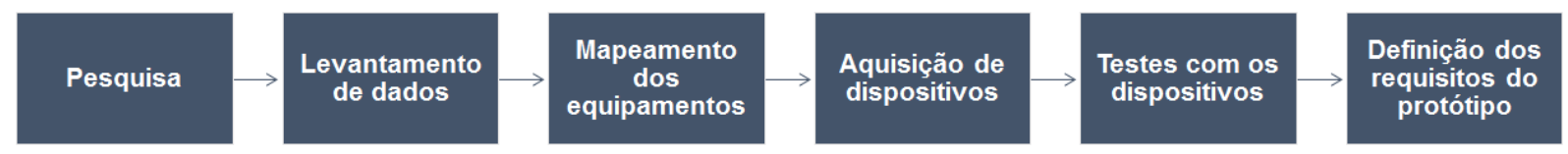

Fonte: Própria, 2019. 
Como mostrado nas etapas metodológicas (Figura 1), mapeou-se equipamentos já existentes que se destinam a inspecionar paredes, indicando ao usuário os materiais que se encontram em seu interior. Em seguida, tais equipamentos foram testados avaliando se suas características e funcionalidades poderiam se adequar à solução dos problemas do caso proposto. Por fim, foram definidas as características e funcionalidades do protótipo de acordo com os resultados obtidos.

\section{RESULTADOS E DISCUSSÃO}

\subsection{Estratégias comuns do furto de energia elétrica e consequências.}

Os métodos mais usuais de fraudes são a adulteração nos medidores e os desvios nas fases antes de medição. No primeiro caso são realizadas alterações no sistema para retardar a rotação do disco responsável por indicar a quantidade de energia consumida. Para que o medidor registre um consumo inferior ao real, são utilizados ímãs ou feitas modificações na eletrônica do equipamento, como abertura de shunts. No caso dos desvios, como ilustrado na Figura 2, a energia passa a ser utilizada na unidade consumidora sem ser contabilizada pelos medidores. [4]

Figura 2. Desvio das três fases antes do sistema de medição. [9]

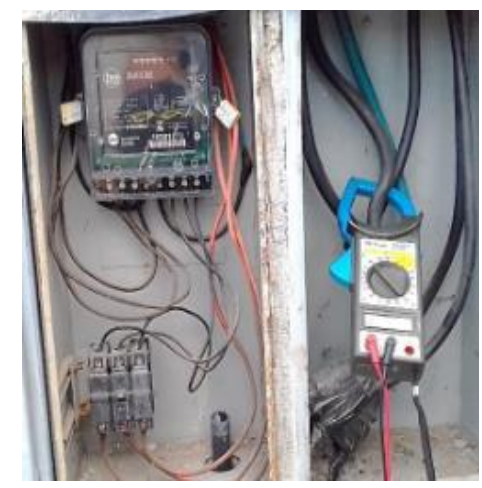

Fonte: Eletrobrás Amazonas, 2016.

Essas fraudes e furtos trazem prejuízos às concessionárias e aos consumidores regulares, abalando a eficiência e a qualidade do serviço prestado, tendo em vista a sobrecarga nas redes elétricas advindas das ligações clandestinas que tornam o sistema de distribuição mais suscetível a interrupções no fornecimento de energia.

\subsection{Metodologia de Detecção Utilizada pelas Concessionárias.}

Para o combate às perdas não técnicas, as concessionárias dispõem de equipes capacitadas para identificação e remoção dos furtos encontrados. As regras para dimensionamento destas equipes e os critérios para cálculo das perdas a recuperar são listados na Resolução Normativa 338/08 da ANEEL, anexo I. [1] 
Tendo como exemplo a Companhia Paulista de Força e Luz (CPFL), a partir dos procedimentos adotados com base nos critérios citados, a distribuidora consegue obter um índice assertivo de apenas $20 \%$ das inspeções realizadas.

Durante as inspeções feitas pela equipe de campo, caso haja suspeita de desvio, são adotados alguns procedimentos de inspeções, existindo, no entanto, alguns fatores que dificultam esta atividade, os quais são: i) a necessidade de desligar o consumidor da rede para realizar a inspeção; ii) a rastreabilidade da inspeção, visto que, não é possível averiguar se esta foi realizada corretamente pelo técnico e; iii) dificuldade na realização do teste do $\mathrm{Kd}$, pois envolve a verificação das medições da potência ativa e da corrente elétrica momentânea, que está sempre variando.

Diante dos padrões estabelecidos, a Figura 3 ilustra uma pesquisa das principais formas de desvios que foram encontrados pela CPFL.

Figura 3. Fraudes mais usuais. [11]

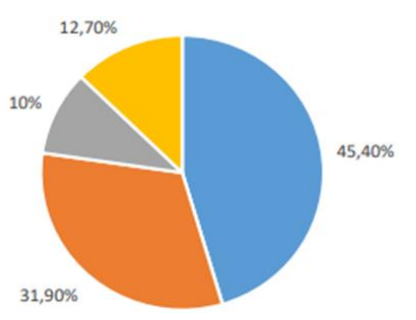

- Na ligação antes da entrada da caixa de medição

- Na ligação do medidor

- Na ligação dentro da caixa de medição

- Nos mecanismos internos do medidor

Fonte: CPFL

De acordo com a Figura 3, é possível notar que os desvios, em sua grande maioria, são feitos antes do sistema de medição, para o qual o protótipo em questão se destina. Para comprovação do furto ou fraude, utiliza-se o procedimento de perícia técnica adotado pelas polícias civis dos estados para comprovar furto ou fraude de energia, gerando provas do ilícito durante a instrução criminal, na fase de inquérito ou durante o processo penal. O código de processo penal estabelece, em seu artigo 169, como deve se proceder a perícia:

Art. 169 - Para o efeito de exame do local onde houver sido praticada a infração, a autoridade providenciará imediatamente para que não se altere o estado das coisas até a chegada dos peritos, que poderão instruir seus laudos com fotografias, desenhos ou esquemas elucidativos.

Embora cada concessionária adote uma metodologia diversa para o combate às perdas não técnicas, poucas fazem uso da perícia técnica para constatação da fraude ou furto. Isto se deve às dificuldades burocráticas que as empresas encontram ao apresentar queixa-crime nas delegacias após a identificação do usuário fraudador. Desta forma, embora pautados em regulamentos ANEEL, onde a concessionária pode multar e interromper o fornecimento ao agente fraudador, não é possível dar prosseguimento ao processo penal, devido à falta de provas que possam ser utilizadas em juízo. Este problema leva à impunidade do agente fraudador, que, inocentado no processo penal por falta de provas, arma-se de ferramentas para acionar judicialmente a concessionária, exigindo reparação por perdas e danos. A dificuldade em condenar os fraudadores potencializa o ambiente favorável à prática e reincidência do crime de furto de energia. 


\subsection{Características levantadas nos equipamentos de mercado.}

Existem dispositivos de mercado capazes de detectar a presença de objetos no interior de paredes. Para análise dos equipamentos que baseiam-se no princípio de See Through Wall, foi feita uma comparação dos mais utilizados no âmbito internacional (Quadro 1), e levantadas as funcionalidades desses.

Quadro 1. Comparação entre Stud Finders do mercado Internacional

\begin{tabular}{|c|c|c|c|c|c|c|c|}
\hline $\begin{array}{l}\text { Matriz de } \\
\text { Comparosăó }\end{array}$ & $\begin{array}{l}\begin{array}{c}\text { Aikotoo } \\
\text { Magnetic }\end{array} \\
\text { 시 }\end{array}$ & $\begin{array}{c}\text { Classware } \\
0\end{array}$ & Multi-scanner & $\begin{array}{c}\substack{\mathrm{CH} \\
\text { Honson }} \\
8\end{array}$ & FS710PRO & DMso4 & 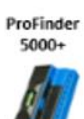 \\
\hline 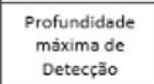 & $0,041 \mathrm{~m}$ & $0,076 \mathrm{~m}$ & $0,051 \mathrm{~m}$ & $0,254 \mathrm{~m}$ & $0,043 \mathrm{~m}$ & $0,050 \mathrm{~m}$ & $0,038 \mathrm{~m}$ \\
\hline $\begin{array}{c}\text { Necessidade de } \\
\text { Bateria }\end{array}$ & $9 \mathrm{~V}$ & $9 \mathrm{~V}$ & $9 \mathrm{~V}$ & $\mathrm{x}$ & Aa & $9 \mathrm{v}$ & AA \\
\hline $\begin{array}{l}\text { Exibição de centro } \\
\text { e borda } \\
\text { simultoneamente }\end{array}$ & $x$ & $\checkmark$ & $x$ & - & $\checkmark$ & - & \\
\hline $\begin{array}{l}\text { Indicador de } \\
\text { Proximidade }\end{array}$ & $x$ & $\checkmark$ & $x$ & $x$ & $\checkmark$ & $x$ & $\checkmark$ \\
\hline
\end{tabular}

Fonte: Elaboração Própria, 2018.

Apesar da característica de detecção de objetos embutidos, os Stud Finders não se mostraram eficientes no que diz respeito à localização precisa e identificação do material detectado de acordo com os dados obtidos. Tal característica é uma limitação, visto que, um detector de desvios embutidos deve ser capaz de distinguir diferentes materiais, como fios e tubulações, e a localização do desvio de forma precisa. Sem esta capacidade a inspeção não pode ser realizada de maneira assertiva.

Além disso, apesar destes equipamentos serem largamente utilizados nos Estados Unidos, a capacidade de penetração das ondas emitidas por estes equipamentos (0,07 m em condições ideais - vide Quadro 1) são insuficientes para aplicações em alvenaria. No caso brasileiro, as paredes a serem inspecionadas são de alvenaria e costumam ter entre 0,12 e 0,17 m de espessura, razão pela qual fica reiterada a inaplicabilidade dos equipamentos de mercado listados no Quadro 1.

Em âmbito nacional são comumente encontrados Wall Scanners que são dispositivos utilizados na construção civil para localizar e identificar o tipo de material detectado no interior de paredes. Dentre os equipamentos mais vendidos, destacaram-se o D-Tect 120, D-Tect 150, da Fabricante Bosch, e o DCT419 da Fabricante DeWalt.

O D-Tect 120 detecta objetos em uma máxima profundidade de 0,12 m [12] e não possui sistema de odometria. Com essa característica, apesar de desempenhar a função de identificação dos objetos embutidos, não há uma referência de localização dos mesmos, o que é uma necessidade relevante para a solução do problema apresentado. O D-Tect 150 possui máxima profundidade de detecção de 0,15 $\mathrm{m}$ [13] e o DeWalt 0,076 m. [14] Então, apesar do sistema de odometria e diferenciação dos 
objetos detectados para o operador, ambos os Wall Scanners não possuem profundidade de detecção satisfatórios para serem destinados à detecção em paredes de alvenaria.

\section{CONCLUSÃO}

Neste documento, foram fundamentadas as perdas técnicas e não técnicas, enfatizando esta última ao ressaltar seus impactos causados sobretudo às concessionárias de energia e aos consumidores. Também, foram discutidos os métodos mais comuns utilizados tanto para fraudar as instalações elétricas, quanto para identificar essas irregularidades, apontando os inconvenientes das técnicas de detecção adotadas atualmente e as dificuldades burocráticas.

A partir disso, pode-se perceber que as técnicas de combate aos furtos não têm sido eficazes para as concessionárias, tornando esta uma área oportuna para propostas de inovação tecnológica, caso deste problema de pesquisa, resultando no desenvolvimento de um equipamento capaz de identificar com precisão e de forma rápida os desvios de energia, evitando prejuízos.

Portanto, com os dados obtidos através do processo de pesquisa, foi possível refinar as necessidades e requisitos do protótipo a ser desenvolvido. Buscou-se a convergência entre as necessidades das concessionárias de energia e as principais características dos equipamentos encontrados no mercado. As dificuldades apresentadas para detecção do desvio e o baixo nível de assertividade em tal ação foram determinantes para escolha da tecnologia a ser empregada no protótipo, dentre todas as tecnológicas classificadas no conceito de See Through Wall (Visão através de paredes).

\section{Agradecimentos}

À Light Serviços de Eletricidade S/A pelo financiamento do projeto de pesquisa e desenvolvimento do protótipo, vinculado ao Programa de P\&D do Setor Elétrico da Agência Nacional de Energia Elétrica (ANEEL).

\section{REFERÊNCIAS}

1 TAHAN, C. M. V.; ARANGO, H. Perdas Técnicas de Energia. [S. I.], 2 set. 2005. Disponível em: <http://www2.aneel.gov.br/arquivos/pdf/perdas_tecnicas_de_energia. pdf.> Acesso em: 26 jul. 2019.

${ }^{2}$ Código Penal. Decreto Lei no 2848 de 07 de dezembro de 1940, Art. 155.

${ }^{3}$ Código Penal. Decreto Lei no 2848 de 07 de dezembro de 1940, Art. 171.

${ }^{4}$ Governo do Brasil. Consumo irregular de energia gera prejuízo de $\mathbf{R} \mathbf{8 , 1}$ bilhões ao ano. 31 mai. 2011. ANEEL. Disponível em: $<$ http://legado.brasil.gov.br/noticias/infraestrutura/2011/05/consumo-irregular-deenergia-gera-prejuizo-de-r-8-1-bilhoes-ao-ano> Acesso em: 23 jul. 2019. 
5 FERREIRA, T. S. D. Método Para Detecção E Localização De Perdas Não Técnicas Em Sistemas De Distribuição Explorando Medidores Inteligentes. 2015. Campinas, SP, 2015. Disponível em: <http://taurus.unicamp.br/bitstream/REP OSIP/259997/1/Ferreira_ThiagoSonnewendDiniz_M.pdf.> Acesso em: 26 jul. 2019.

${ }^{6}$ ANEEL. Revisão Tarifária Periódica das Concessionárias de Distribuição. 06 mai. 2015. Disponível em: <http://www2.aneel.gov.br/arquivos/PDF/Subm\%c3\%b3du lo\%202.6_V3.pdf.> Acesso em: 23 jul. 2019.

7 ANEEL. Perdas de Energia. 25 nov. 2015. Disponível em: $<$ http://www.aneel.gov.br/metodologia-distribuicao/-/asset_publisher/e2INtBH4EC4e/ content/perdas/654800?inheritRedirect=false. $>$ Acesso em: 23 jul. 2019.

${ }^{8}$ C. A. d. S. PENIN. Combate, Prevenção e Otimização das Perdas Comerciais de Energia Elétrica. p. 227, 2008.

${ }^{9}$ ELETROBRÁS AMAZONAS. Empresa de Reciclagem Flagrada com Furto de Energia. 1 set. 2016. Disponível em: <http://www.eletrobrasamazonas.com/cms/inde x.php/empresa-de-reciclagem-flagrada-com-furto-de-energia/>. Acesso em: 23 jul. 2019.

10 ANEEL. Resolução Normativa que dispõe sobre as metodologias para o $2^{\circ}$ ciclo de revisão tarifária periódica da distribuição. 2008. Disponível em: <http://www2.aneel.gov.br/cedoc/ren2008338.pdf>. Acesso em: 22 jul. 2019.

11 CPFL. Seleção De Projetos De Pesquisa, Desenvolvimento Tecnológico E Inovação (PD\&I) Nos Segmentos De Geração, Transmissão E Distribuição De Energia Elétrica. 2018. Disponível em: <https://www.cpfl.com.br/energiassustentaveis/inovacao/chamadapublica/Documents/EDITAL_TEMA\%2001_PERDAS \%20N\%C3\%830\%20TECNICAS.pdf>. Acesso em: 22 jul. 2019.

12 BOSCH. D-tect 120 Professional. 2016. Disponível em: <https://www.boschprofessional.com/binary/ocsmedia/optimized/full/o245345v21_160992A212_201610. pdf>. Acesso em: 22 jul. 2019

$13 \mathrm{BOSCH}$. D-tect 150 Professional. 2018. Disponível em: <https://www.boschprofessional.com/binary/ocsmedia/optimized/full/o293576v21_160992A4G0_201808. pdf>. Acesso em: 22 jul. 2019.

14 DEWALT. DCT419S1 HAND HELD WALL SCANNER. 2018. Disponível em: $<$ https://www.dewalt.com/products/power-tools/lasers-and-instruments/hand-heldwall-scanner/dct419s1>. Acesso em: 23 jul. 2019. 